\title{
Manometric changes of the esophagus in morbidly obese patients
}

\author{
ANDRADA L. POPESCU ${ }^{1,2}$, RALUCA S. COSTACHE ${ }^{1,2}$, DANIEL O. COSTACHE ${ }^{3}$, \\ VASILE D. BALABAN ${ }^{1,4}$, MARIANA JINGA ${ }^{1,2}$, FLORENTINA IONITA-RADU ${ }^{2,5}$, \\ ANA CARUNTU $^{6}$ and CARMEN FIERBINTEANU-BRATICEVICI ${ }^{1,7}$
}

\begin{abstract}
${ }^{1} 5$ th Clinical Department, Gastroenterology and Internal Medicine Discipline, 'Carol Davila' University of Medicine and Pharmacy, 050474 Bucharest; Departments of ${ }^{2}$ Gastroenterology, ${ }^{3}$ Research, and ${ }^{4}$ Ambulatory Department, 'Carol Davila' University Central Emergency Military Hospital, 010825 Bucharest; ${ }^{5}$ Faculty of Medicine, 'Titu Maiorescu' University, 040441 Bucharest; ${ }^{6}$ Department of Maxillofacial Surgery, 'Carol Davila' University Central Emergency Military Hospital, 010825 Bucharest; ${ }^{7}$ Gastroenterology Department, Emergency University Hospital, 050098 Bucharest, Romania
\end{abstract}

Received February 1, 2021; Accepted March 3, 2021

DOI: $10.3892 / \mathrm{etm} .2021 .10036$

\begin{abstract}
This prospective study aimed to determine the manometric pattern and the prevalence of esophageal dysmotility in 79 morbidly obese patients selected for laparoscopic sleeve gastrectomy. After clinical evaluation and upper gastrointestinal endoscopy,high-resolutionesophageal manometry was performed. The esophageal peristalsis, lower esophageal sphincter (LES) basal pressure, and LES relaxation were evaluated. Demographic data showed a predominance of females (55.70\%) and both females and males were in the 5 th decade of life. In addition, approximately $3 / 4$ of the patients (78.48\%) were from the urban zone. The mean body mass index of the patients was $46.40 \pm 6.0069 \mathrm{~kg} / \mathrm{m}^{2}$, with a maximum of $61 \mathrm{~kg} / \mathrm{m}^{2}$. The LES basal pressure was normal in $59.49 \%$ of the patients, with a mean value of $31.40 \pm 18.43 \mathrm{mmHg}$. LES basal hypertonia was observed in $26.58 \%$, and LES hypotonia in $13.93 \%$ of patients; $46.84 \%$ (37 patients) had abnormal manometric findings: $24.05 \%$ (19 patients) had EGJ outflow obstruction, $12.66 \%$ (10 patients) ineffective esophageal motility, $3.8 \%$ (3 patients) distal esophageal spasm, 3.8\% (3 patients) Jackhammer esophagus, 2 cases were suggestive for type 2 achalasia but in asymptomatic patients. Ineffective esophageal motility was not associated with diabetes mellitus type 2 or erosive esophagitis according to our data. Hiatal hernia $(\mathrm{HH})$ was manometrically diagnosed in 23 patients (29.11\%). Preoperative high-resolution esophageal manometry in obese patients demonstrated a high prevalence of motility disorders, but in asymptomatic patients, thus in the future, we require more studies and larger cohorts to better appreciate the clinical impact.
\end{abstract}

Correspondence to: Dr Raluca S. Costache, Department of Gastroenterology, 'Carol Davila' University Central Emergency Military Hospital, 134 Calea Plevnei Street, 010825 Bucharest, Romania

E-mail: raluca.costache@umfcd.ro

Key words: obesity, esophageal manometry, LES pressure, esophageal dysmotility, ineffective esophageal motility

\section{Introduction}

Obesity is a common and costly disease, defined as abnormal or excessive fat accumulation, usually caused by excessive food intake, genetic susceptibility, and lack of physical activity. The body mass index or BMI (weight in kilograms divided by the square of height in meters) is used in clinical practice to classify adults as overweight $(\mathrm{BMI} \geq 25)$ or obese $(\mathrm{BMI} \geq 30)(1)$.

The World Health Organization (WHO) warns that obesity has nearly tripled since 1975 . Once considered a high-income country problem, overweight and obesity are now increasing in low- and middle-income countries, especially in urban zones. Worldwide, $39 \%$ of adults were estimated to be overweight in 2016 , and $13 \%$ were obese (1). Obesity in the adult population in Romania was estimated at $9.4 \%$ in 2014, compared to a European average of $15.9 \%$ (2).

Obesity is a major risk factor for diabetes, cardiovascular diseases, obstructive sleep apnea, non-alcoholic fatty liver disease, osteoarthritis, social stigmatization, and even cancer $(1,3,4)$.

Literature has focused on the connection between obesity and gastroesophageal reflux disease (GERD), but there are limited data available on esophageal motility in obese individuals, and usually on small cohorts of patients (5). Obesity is a potential risk factor for GERD and the associated complications including erosive esophagitis, Barrett esophagus, and esophageal adenocarcinoma as compared to individuals with a normal BMI (6). Few studies in the literature have examined motility disorders among the morbidly obese population outside the context of GERD; most of them focusing on the relationship between lower esophageal sphincter (LES) pressure and reflux disease (7).

\section{Patients and methods}

We performed a prospective study from January 2017 to July 2020, with a cohort of 79 morbidly obese subjects submitted for vertical laparoscopic gastrectomy who were evaluated with high-resolution esophageal manometry before the surgical intervention, additional to upper gastrointestinal 
tract endoscopy and clinical assessment (GERD-HRQL questionnaire). The objective was to better understand the manometric profile of obesity, especially regarding esophageal peristalsis, LES basal pressure, and LES relaxation.

This is part of a larger study approved by the Ethics and Research Committee of the Carol Davila University Central Emergency Military Hospital Bucharest, designed to observe and reduce the prevalence of upper gastrointestinal symptoms and complications after laparoscopic sleeve gastrectomy. All patients agreed to be included in the study and signed the informed consent. The study exclusion criteria were: Other bariatric procedures (surgical or endoscopically), previous gastric surgery, patients not willing to sign the informed consent, severe bleeding disorders, inability to tolerate nasal intubation, or esophageal obstruction preventing passage of the manometric catheter.

High-resolution esophageal manometry is the current state-of-the-art diagnostic tool to evaluate esophageal motility (8). The Chicago Classification version 3.0 is the latest published esophageal motility disorder classification by The International High-Resolution Manometry Working Group, based on high-resolution esophageal pressure topography, and represents the standard interpretation scheme used in clinical practice (9).

High-resolution esophageal manometry (HRM) was performed with a 36-channel High-Resolution Solid-State Catheter Sierra, by a single examiner, after $6 \mathrm{~h}$ of fasting and a previous upper gastrointestinal endoscopy following the standard protocol: a baseline phase for resting pressure and a series of 10 wet swallows of $5 \mathrm{ml}$ of water in the supine position with the interval of a minimum $30 \mathrm{sec}$ between swallows (10). There were no complications during the exams.

Drugs that may affect esophageal motility were discontinued for at least $48 \mathrm{~h}$ before the examination (nitrates, calcium channel blockers, antiemetics, prokinetics, $\mathrm{H} 2$ blockers, antidepressants).

HRM data were analyzed according to the Chicago Classification version 3.0, using the ManoView ESO software program (a component of the ManoScan system), providing visual images and quantitative physiological parameters. The manometric data from the obese cohort focused on analyzing the LES basal pressure, the deglutitive LES relaxation (residual pressure), esophageal peristalsis, and contractility pattern.

The GERD-HRQL questionnaire was used as a quantitative method for measuring symptom severity of GERD in morbidly obese. The scale has 15 questions concerning heartburn, regurgitation, pain, and dysphagia; each item is scored from 0 to 5 , and the $16^{\text {th }}$ item is a subjective appreciation of the present clinical condition. The GERD-HRQL is easy and fast, it takes only a few minutes to complete, and recently is used also to measure the quality of life of morbidly obese patients (11). The total score was calculated by summing the individual scores to questions 1-15, with a highest possible score (worst symptoms) of 75, and lowest possible score 0 . The heartburn score was obtained by summing the individual scores to questions $1-6$, and the regurgitation score summing 10-15 responses; scores of $\leq 12$ with each question not exceeding 2 indicate heartburn or regurgitation elimination (12).
Statistical analysis. Data are presented as a percentage from the total (\%), mean \pm standard deviation (range min-max, where applicable). Statistical analysis was carried out using SPSS Statistics 25 (IBM Corp.). Descriptive statistics included measures of central tendency for quantitative variables, counts, and percentages for categorical variables and corresponding graphs.

\section{Results}

In our study population, females were predominant [55.70\% (44/79 patients)], with 2 peaks of age at $45-50$ years and 60 years, probably linked to menopause and hormonal changes. For men, there were also 2 frequency peaks at 35-40 years and another one at 50 years. The youngest patient was a 19-year of age female, struggling from childhood with excess fat, who previously tried a restrictive diet recommended by the nutritional doctor, as well as physical exercise, and anorexigenic drugs, and currently she was proposed for bariatric surgery. Unfortunately, obesity is increasing also in children not only in adults, and most of them experience social isolation and stigmatization, anxiety, and low self-esteem (13).

Approximately $3 / 4$ of the patients $(78.48 \%$ ) were from the urban zone, and the mean BMI was $46.40 \pm 6.0069 \mathrm{~kg} / \mathrm{m}^{2}$, with a maximum of $61 \mathrm{~kg} / \mathrm{m}^{2}$.

The mean LES basal pressure was $31.40 \pm 18.43 \mathrm{mmHg}$. The LES basal pressure was normal in $59.49 \%$ of the patients; LES basal hypertonia was observed in $26.58 \%$, and LES hypotonia in $13.93 \%$ of the patients. LES hypotonia may predispose to GERD, but in our cohort, there was no correlation between LES low pressure and GERD and erosive esophagitis, based on reflux symptomatology and upper gastrointestinal endoscopy; yet, one study limitation was the absence of 24-h esophageal $\mathrm{pH}$ monitoring.

The mean integrated relaxation pressure (IRP) was $9.75 \pm 10.71 \mathrm{mmHg}$, with a maximum of $38 \mathrm{mmHg}$. When the IRP value was high $(>15 \mathrm{mmHg}$ according to the Sierra catheter specifications), we further analyzed the esophageal contractility to establish a diagnosis of achalasia or esophagogastric junction (EGJ) outflow obstruction using the Chicago Classification v3.0.

The esophageal contractility was assessed with distal contractile integral (DCI) expressed in $\mathrm{mmHg}-\mathrm{sec}-\mathrm{cm}$, which means amplitude $\mathrm{x}$ duration $\mathrm{x}$ length of the contractile wave induced by wet deglutition test. Mean DCI was $2,693.12 \pm 1,919.31 \mathrm{mmHg}$-sec-cm), with a minimum of $249.7 \mathrm{mmHg}$-sec-cm and a maximum of $9,373.5 \mathrm{mmHg}$-sec-cm; the normal values admitted for DCI range between 450 and $8,000 \mathrm{mmHg}$-sec-cm.

The distal latency (DL) used to identify premature contraction (value $<4.5 \mathrm{sec}$ ) had a mean value of $6.38 \pm 1.16 \mathrm{sec}$ (minimum 4.0, maximum of $9.8 \mathrm{sec}$ ). When more than 2 swallows are premature, with normal IRP the diagnose is distal esophageal spasm.

From the obese study group, $46.84 \%$ (37/79 patients) had abnormal manometric findings: 24.05\% (19/79 patients) had EGJ outflow obstruction, $12.66 \%$ (10/79 patients) ineffective esophageal motility (IEM), 3.8\% (3 patients) distal esophageal spasm (SED), 3.8\% (3 patients) hypercontractile esophagus Jackhammer esophagus, and 2 cases were suggestive for 


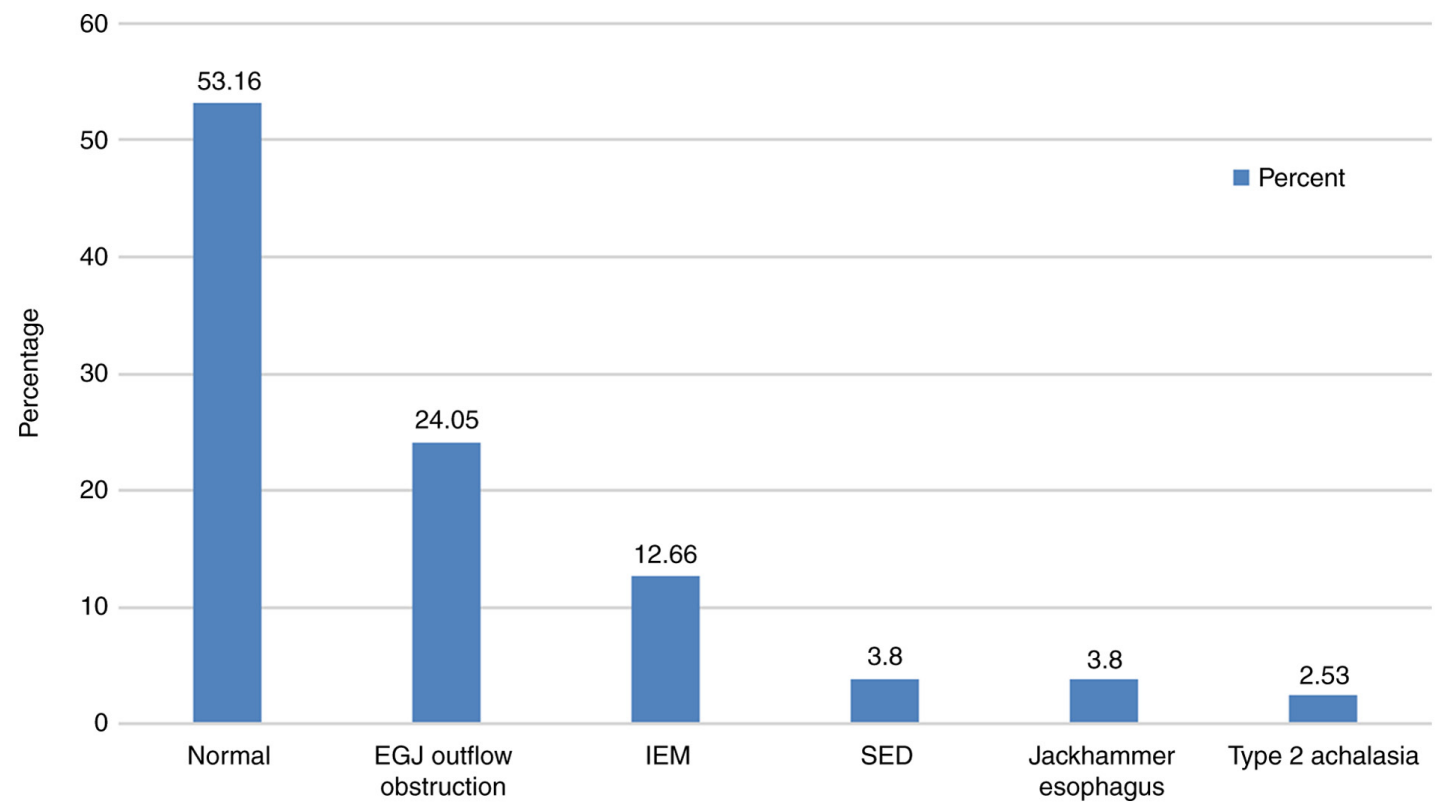

Figure 1. Chicago v3.0 diagnostic distribution of the morbidly obese patients. EGJ, esophagogastric junction; IEM, ineffective esophageal motility; SED, distal esophageal spasm.

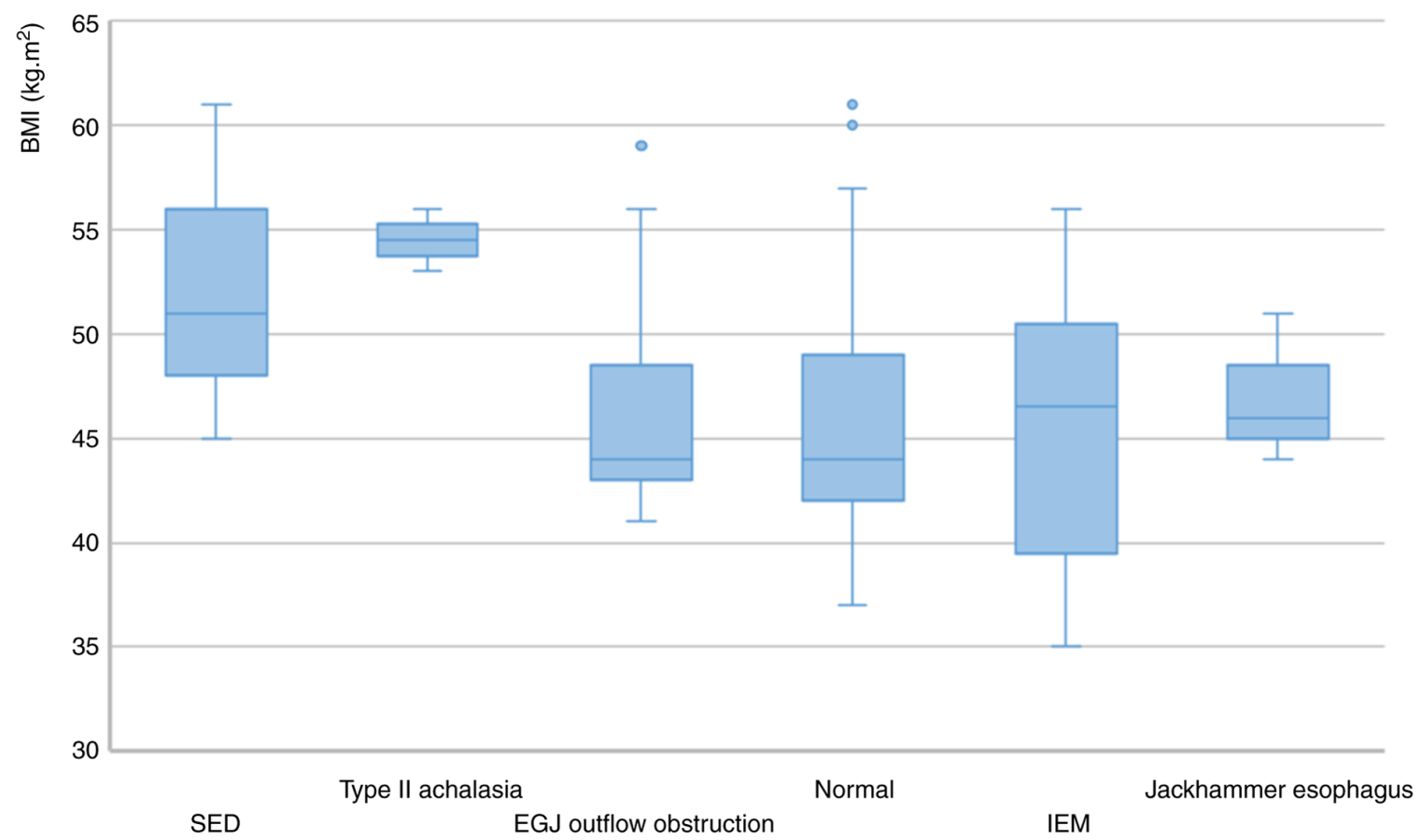

Figure 2. Correlation between Chicago v3.0 diagnostic and body mass index (BMI). EGJ, esophagogastric junction; IEM, ineffective esophageal motility; SED, distal esophageal spasm.

type 2 achalasia $(2.53 \%)$ but in asymptomatic patients ( 0 points Eckardt score) (Fig. 1). Ineffective esophageal motility was not associated with diabetes mellitus type 2 or erosive esophagitis according to our data.

To ascertain whether there is a correlation between the grade of obesity and specific manometric findings, we analyzed the BMI values for each manometric diagnosis, and the data that we obtained are provided in Fig. 2. Considering the small study cohort, a conclusion on this topic was difficult. Yet, the research concept can be further materialized as a research idea in the future, on a larger cohort, to see whether we can find a model of prediction based on BMI values.

From the total 79 obese patients evaluated for sleeve gastrectomy, only 6 of them had significant GERD symptoms (with regurgitation score $>12$ points, heartburn score $>12$ points on the GERD-HRQL questionnaire). The heartburn score was calculable (different from zero) in 19 patients $(24.05 \%$ ), with an average of 7.89 points; the regurgitation score was similar with an average of 8.94 points. A positive score for dysphagia ( $>2$ points) was obtained for 3 patients, while for 
pain 2 patients. However, 16 patients $(20.25 \%)$ had erosive esophagitis on upper gastrointestinal endoscopy (12 grade A and 4 grade $\mathrm{B}$ according to Los Angeles classification), even if only mild reflux symptoms were present or the patients were asymptomatic. Physiopathologicaly GERD may be related to the presence of $\mathrm{HH}$, and in our obese group, the prevalence pof HH was high (29.11\%).

\section{Discussion}

The present study aimed to determine the manometric pattern and the prevalence of esophageal dysmotility in morbidly obese patients. In our obese study population, we observed a female predominance and high frequency in the five decades of life in both female and male groups. In additon, the patients were predominantly from urban regions, in accordance with the literature due to fast-food consumption, a high-calorie diet, and lack of physical exercise (14).

The prevalence of manometric abnormalities in the morbidly obese population was high and included LES basal pressure alteration, trouble in LES relaxation, disturbance in esophageal body contractility (ineffective esophageal motility, hypercontractile esophagus, distal esophageal spasm); yet, the patients were mostly asymptomatic, thus this may be an accidental finding or support to the idea that morbidly obese patients may have abnormal visceral sensation-not always a predictor of the post gastrectomy evolution (15).

It is known that obese people are predisposed to GERD (16), $\mathrm{HH}$ being one of the risk factors (17). HH is usually more frequent in obese individuals because of pressure modification at the LES due to increased intraabdominal pressure and visceral fat (18). HH was manometrically diagnosed in 23 obese patients (29.11\%); 16 patients had erosive esophagitis on upper GI tract endoscopy, although only 6 patients had significant GERD symptoms on the GERD-HRQL questionnaire. This might be explained by a high sensitivity threshold of obese to GERD symptoms. One study limitation is the absence of 24-h esophageal $\mathrm{pH}$ monitoring. The presence of $\mathrm{HH}$ is not a contraindication for laparoscopic sleeve gastrectomy, but it is recommended to be repaired in the same surgical intervention (19).

Esophageal manometry remains an important tool for the study of LES continence and esophageal body function and should be implemented in the evaluation of morbidly obese patients before bariatric surgery, at least in patients with reflux symptoms or esophagitis (20). If a patient presents reflux symptoms and manometry confirms an incompetent LES, sleeve gastrectomy is probably not the best option and Roux-en-Y gastric bypass might be a better choice (19). By assessing esophageal motility, high-resolution manometry helps to select those patients who could develop postoperative esophageal motor complications (21).

\section{Acknowledgements}

Not applicable.

\section{Funding}

No funding was received for preparation of this article.

\section{Availability of data and materials}

Additional information concerning the present study is available from the corresponding author upon reasonable request.

\section{Authors' contributions}

ALP performed the examination, and was responsible for the conception and design of the work, and writing of the first draft. RSC was responsible for data acquisition, analysis, and interpretation of data. DOC was responsible acquisition, analysis, interpretation of data for the work, and verifying the final draft. VDB performed the statistical analysis. MJ carried out the critical revision for important intellectual content. FIR was responsible for conception and design of the work. AC was responsible for acquisition, analysis, interpretation of data for the work. CFB carried out the final check for all the data and the final approval of the version to be published. All authors read and approved the final manuscript.

\section{Ethics approval and consent to participate}

This is part of a larger study approved by the Ethics and Research Committee of the 'Carol Davila' University Central Emergency Military Hospital Bucharest. All patients agreed to be included in the study and signed the informed consent. All patients agreed to be included in the study and signed the informed consent.

\section{Patient consent for publication}

Not applicable.

\section{Competing interests}

The authors declare that they have no competing interests.

\section{References}

1. WorldHealthOrganization:Obesityandoverweight.https://www. who.int/news-room/fact-sheets/detail/obesity-and-overweight. Acecessed April 1, 2020.

2. Popa AR, Fratila O, Rus M, Aron RA, Vesa CM, Pantis C, Diaconu CC, Bratu O, Bungau S and Nemeth S: Risk factors for adiposity in the urban population and influence on the prevalence of overweight and obesity. Exp Ther Med 20: 129-133, 2020.

3. Zaha DC, Vesa C, Uivarosan D, Bratu O, Fratila O, Tit DM, Pantis C, Diaconu CC and Bungau S: Influence of inflammation and adipocyte biochemical markers on the components of metabolic syndrome. Exp Ther Med 20: 121-128, 2020.

4. Eurostat: European Health Interview Survey: Almost 1 adult in 6 in the EU is considered obese. Share of obesity increases with age and decreases with education level. 203, 2016. https://ec.europa. eu/eurostat/documents/2995521/7700898/3-20102016-BP-EN. pdf/c26b037b-d5f3-4c05-89c1-00bf0b98d646. Accessed October 20, 2016.

5. Corley DA and Kubo A: Body mass index and gastroesophageal reflux disease: A systematic review and meta-analysis. Am J Gastroenterol 101: 2619-2628, 2006.

6. Hampel H, Abraham NS and El-Serag HB: Meta-Analysis: Obesity and the risk for gastroesophageal reflux disease and its complications. Ann Intern Med 143: 199-211, 2005.

7. Koppman J, Poggi L, Szomstein S, Ukleja A, Botoman A and Rosenthal R: Esophageal motility disorders in the morbidly obese population. Surg Endosc 21: 761-764, 2007.

8. Yadlapati R: High-Resolution esophageal manometry: Interpretation in clinical practice. Curr Opin Gastroenterol 33: 301-309, 2017. 
9. Bredenoord AJ, Fox M, Kahrilas PJ, Pandolfino JE, Schwizer W, Smout AJ; International High Resolution Manometry Working Group: Chicago classification criteria of esophageal motility disorders defined in high resolution esophageal pressure topography (EPT). Neurogastroenterol Motil 24: 57-65, 2012.

10. Weingart V, Lambertz J and Allescher HD: High-Resolution manometry of the esophagus - Pictorial Atlas and Textbook. Standard Light GmbH, 2012-2013.

11. Guan XL and Wang H: Quality of life scales for patients with gastroesophageal reflux disease: A literature review. Int Journal of Nursing Sciences 2: 110-114, 2015.

12. Hunter JG, Trus TL, Branum GD, Waring JP and Wood WC: A physiologic approach to laparoscopic fundoplication for gastroesophageal reflux disease. Ann Surg 223: 673-685, 1996.

13. Puhl RM and Heuer CA: Obesity stigma: Important considerations for public health. Am J Public Health 100: 1019-1028, 2010.

14. Nurwanti E, Hadi H, Chang JS, Chao JJ, Paramashanti BA, Gittelsohn J and Bai CH: Rural-Urban differences in dietary behavior and obesity: Results of the riskesdas study in 10-18-year-old Indonesian children and adolescents. Nutrients 11: $2813,2019$.
15. Jaffin BW, Knoepflmacher P and Greenstein R: High prevalence of asymptomatic esophageal motility disorders among morbidly obese patients. Obes Surg 9: 390-395, 1999.

16. El-Serag H: The association between obesity and GERD: A review of the epidemiological evidence. Dig Dis Sci 53: 2307-2312, 2008

17. El-Serag HB, Graham DY, Satia JA and Rabeneck L: Obesity is an independent risk factor for GERD symptoms and erosive esophagitis. Am J Gastroenterol 100: 1243-1250, 2005.

18. Khan A, Kim A, Sanossian C and Francois F: Impact of obesity treatment on gastroesophageal reflux disease. World J Gastroenterol 22: 1627-1638, 2016.

19. Garg H, Vigneshwaran B, Aggarwal S and Ahuja V: Impact of concomitant laparoscopic sleeve gastrectomy and hiatal hernia repair on gastro-oesophageal reflux disease in morbidly obese patients. J Minim Access Surg 13: 103-108, 2017.

20. Braghetto I, Lanzarini E, Korn O, Valladares H, Molina JC and Henriquez A: Manometric changes of the lower esophageal sphincter after sleeve gastrectomy in obese patients. Obes Surg 20: 357-362, 2010.

21. Popescu AL, Costache RS, Bucurica S, Patrasescu M Balaban VD, Savulescu FA, Jinga M, Ionita-Radu F and Fierbinteanu-Braticevici C: Laparoscopic sleeve gastrectomy and esophageal motility. Rom J Mil Med 128: 190-195, 2020. 